\title{
Heat and mass transfer within a vertical pipe with a surface heating element of variable size
}

\author{
Antigoni Kleanthous ${ }^{a, b}$ and Robert A. Van Gorder ${ }^{a *}$ \\ ${ }^{a}$ Mathematical Institute, University of Oxford, Andrew Wiles Building, Radcliffe Observatory Quarter, Woodstock Road, Oxford OX2 6GG United Kingdom \\ ${ }^{b}$ Department of Mathematics, University College London, Gower Street, London WC1E 6BT United Kingdom \\ *Robert.VanGorder@maths.ox.ac.uk
}

\begin{abstract}
Heat and mass transfer due to upstream fluid flow in a vertical pipe which is heated in some region due to an external heating element on the surface of the pipe is considered. Unlike most studied in the literature which consider heating uniformly over the entire pipe, we allow for the heater to act over a smaller sub-region of the pipe surface. We first derive a heat and mass transfer model to describe the velocity, pressure, and temperature evolution in a vertical pipe under the assumption of cylindrical symmetry. Using a finite element method we are able to obtain numerical simulations to this model. We compare solutions under a variety of different heater configurations, in order to understand how the size and placement of the heating element on the surface of the pipe will modify the thermal properties of the fluid. We find that a smaller heating element placed near the top of the pipe can still deliver sufficient heat so that the temperature of fluid exiting the top of the pipe has desirable thermal properties for a specific application, and in such cases it is not necessary to heat the entire length of the pipe. Such a configuration could be more efficient, as it requires less material for the heating element, while also requiring less energy for the heating. On the other hand, if the heating element is too small, or poorly placed along the pipe, then it may not be possible to obtain desirable thermal properties in the fluid that would have been possible with a heating element covering the entire pipe length.
\end{abstract}

Keywords: vertical pipe flow, heat transfer, heating element, finite element method

\section{Introduction}

The upward flow of a fluid in a duct or pipe which is heated has been the topic of many studies. Single phase flow is defined as regular flow of a single fluid of a single phase along some domain. Regular flow in a pipe has been studied by Avila et al. [1] to establish when transition to turbulent flow occurs. The effects of a heating element applied in pipe flow with a part of the pipe being insulated have been studied by Faghri et al. [2] through numerical simulations. A similar case where the heating was applied by a step change was studied by Bilir [3], again through numerical simulations. Bernier and Baliga [4] performed a numerical investigation of conjugate conduction and laminar mixed convection in vertical pipes for upward flow and uniform wall heat flux. One application of such numerical studies would be to boreholes in vertical ground heat exchangers [5]. Turbulence under $k-\epsilon$ models was numerically studied by Hiroaki et al. [6] for the forced and natural convection heat transfer in an upward flow due to a uniformly heated vertical pipe.

There have been some analytical studies on the upward flow of a fluid in a uniformly heated pipe as well, often for the case where the flow is assumed one-dimensional. An exact solution for the problem of laminar convective flow under a pressure gradient along a vertical pipe in the case where the walls of the pipe are heated uniformly (under the assumption that velocity and buoyancy profiles far from the pipe entrance do not change with height, and entry-length effects are ignored) was given by Morton [7]. In that study, it was shown that when an upflow is heated the velocity near the walls is increased and that near the axis decreased until, for sufficiently large Rayleigh numbers, definite velocity and thermal boundary layers are formed. An exact solution was given by Gupta [8] for the problem of unsteady laminar convective flow under a pressure gradient along a vertical pipe which was heated uniformly. Barletta and Lazzari [9] analytically study fully developed mixed convection flow in a vertical circular duct assuming laminar parallel flow. In this study, the wall heat flux is assumed uniform in the axial direction yet depends on the angular variable, so that a proportion of the pipe can be viewed as heated and insulated. Smith et al. [10] analytically studied the temperature distribution in laminar pipe flow with a step change in wall heat flux.

Despite all of the previously mentioned interest in vertical pipe flow within a heated pipe, there has been little work on partially heated pipes, that is, pipes which are heated symmetrically only over a segment on the pipe. Applications might include coffee makers $[11,12]$, nuclear reactors, refrigerator systems [13], and thermosyphons [14], for which it may be adventagious or more efficient to heat only a segment of the pipe rather than the entire length of the pipe. Motivated by this, in this paper we shall consider upward fluid flow within a pipe, in the case where only a cylindrical segment of the pipe is heated. The system of interest consists of a cylindrical pipe of length $L$ 
that exerts some electrical push $\mathbf{F}_{\text {meter }}$ in the blue region and the red region $(a \leq z \leq b)$ represents heat applied on the boundary, as in Figure 1. Note that the meter may be calibrated to give pulses. Pulsating heat pipes have been studied before in some contexts, but again under the assumption of uniform heating. For instance, Charoensawan et al. [15] consider pulsating heat pipes with the entire apparatus submerged in a heat bath which provides the heating. In addition to the applications listed above, recent applications of heated pipe flow to nanofluids have also been considered [16, 17, 18].

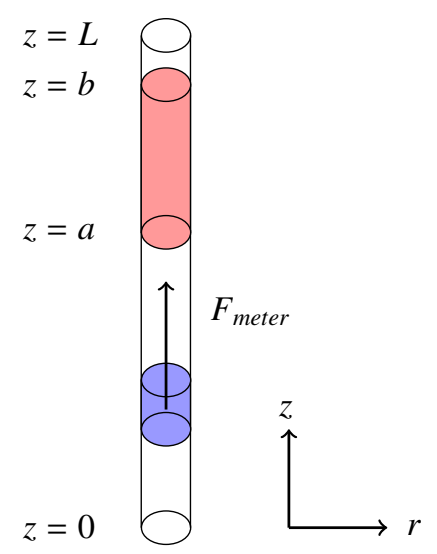

Figure 1: Schematic representation of the system.

For the development of our mathematical model, we choose to consider the case of single phase flow, corresponding to the case where a heater is applied in some region of the pipe, heating the fluid, but never reaching saturation temperature where evaporation begins. This is particularly useful in applications where a fluid is being heated but no boiling or transition to other phases will occur or are desired. In the case of a boiler (rather than a heater) placed on the pipe, there will be a phase transition, and a multiphase model will be needed. We consider this case in a companion paper [19], where we study a one-dimensional model for a boiler placed along a cylindrical subsection of a larger cylindrical pipe.

The paper is organized as follows. In Section 2 we derive a heat and mass transfer model under the assumption of cylindrical geometry, and then we provide a non-dimensionalisation of this model. In Section 3 we give the numerical solution approach (a finite element method), and we give numerical results and discuss them in Section 4, in order to deduce the influence of the heater on the vertical pipe flow. We give concluding remarks in Section 5.

\section{Derivation of the heat and mass transfer model}

We assume incompressible, Newton flow of density $\rho$, viscosity $\mu$, and velocity field $\mathbf{u}=(u(r, \theta, z), v(r, \theta, z), w(r, \theta, z))$ in the system of Figure 1. We assume that the temperature never passes the critical point where evaporation begins, and therefore no phase changes of the fluid occur.
To model the system we use the Boussinesq approximation [20] as in [21] and ignore any variations other than the density variation. The Boussinesq approximation is only valid when the difference in density $\Delta \rho$ is small compared to some constant density $\rho_{0}$, in this case, the density of the fluid at the initial temperature. This holds in our case, as we are modelling flow of water or similar fluids. Variations of the density are ignored, except for when they give rise to gravitational force. Therefore, the continuity equation

$$
\frac{\partial \rho}{\partial t}+\nabla \cdot(\rho \mathbf{u})=0
$$

becomes

$$
\nabla \cdot \mathbf{u}=0
$$

In the momentum equation

$$
\rho \frac{D \mathbf{u}}{D t}=-\nabla p+\mu \nabla^{2} \mathbf{u}+\mathbf{F},
$$

we replace $\rho$ by $\rho_{0}$. The term $\mathbf{F}$ represents the contribution of any forces that act on a volume element of fluid. In this case, we have the force from the meter $\mathbf{F}_{\text {meter }}$ and a force for the effect of gravity

$$
\mathbf{F}_{g}=\rho \mathbf{g}
$$

where the gravitational acceleration is derivable from a potential

$$
\mathbf{g}=-\nabla \Phi
$$

In this case, we expect the variations in density to be important and therefore let

$$
\rho=\rho_{0}+\Delta \rho .
$$

Therefore,

$$
\mathbf{F}_{g}=-\left(\rho_{0}+\Delta \rho\right) \nabla \Phi,
$$

which implies

$$
\mathbf{F}_{g}=-\nabla\left(\rho_{0} \Phi\right)+\Delta \rho \mathbf{g}
$$

Letting $P=p+\rho_{0} \Phi$ and substituting into the momentum equation we obtain

$$
\rho_{0} \frac{D \mathbf{u}}{D t}=-\nabla P+\mu \nabla^{2} \mathbf{u}+\Delta \rho \mathbf{g}+\mathbf{F}_{\text {meter }}
$$

The reason why we can treat $\rho$ as a constant except in the term involving gravitational forces is that the term $\Delta \rho \mathbf{g}$ can produce significant effects even when $\Delta \rho / \rho_{0} \ll 1$ [21]. In this case, we linearize the dependence of $\rho$ on the temperature $T$

$$
\Delta \rho=-\alpha \rho_{0} \Delta T
$$

where $\alpha$ is the coefficient of expansion of the fluid. We therefore obtain the Boussinesq equation

$$
\frac{D \mathbf{u}}{D t}=-\frac{1}{\rho} \nabla p+\nu \nabla^{2} \mathbf{u}-\alpha \Delta T \mathbf{g}+\frac{1}{\rho} \mathbf{F}_{\text {meter }},
$$


where we have renamed $\rho_{0}$ into $\rho$ and $P$ into $p$, and $v=\mu / \rho$ is the kinematic viscosity. The term $-\alpha \Delta T \mathbf{g}$ represents the buoyancy force.

We now need a temperature equation. We further assume that the fluid has a constant heat capacity per unit volume, $\rho C_{p}$. Then

$$
\rho C_{p} \frac{D T}{D t}=\text { rate of heating per unit volume of fluid. }
$$

This heating is the result of heat transferred from neighbouring fluid particles by thermal conduction. The conductive heat flux is

$$
\mathbf{H}=-k \nabla T
$$

where $k$ is the thermal conductivity of the fluid. Therefore

$$
\rho C_{p} \frac{D T}{D t}=-\nabla \cdot \mathbf{H}+J
$$

where $J$ is the rate of internal heat generation per unit volume. Taking $k$ to be constant, and defining the thermal diffusivity as $\kappa=k / \rho C_{p}$, we obtain

$$
\frac{\partial T}{\partial t}+(\mathbf{u} \cdot \nabla) T=\kappa \nabla^{2} T+\frac{J}{\rho C_{p}} .
$$

The term $(\mathbf{u} \cdot \nabla) T$ is the advection term and represents the transport of heat by the motion, while $\kappa \nabla^{2} T$ is the conduction term and $J / \rho C_{p}$ the heat generation term.

Equations (2), (11) and (15) are the basic equations of convection in the Boussinesq approximation.

Considering now the geometry of the system and assuming axisymmetric flow in cylindrical coordinates, the velocity field is of the form $\mathbf{u}=(u(t, r, z), w(t, r, z))$. As the pipe is vertical, the force due to gravity and the force due to the meter $\mathbf{F}_{\text {meter }}$ are directed along the $z$ axis, and therefore $\mathbf{g}=(0, g)$ and $\mathbf{F}_{\text {meter }}=$ $\left(0, F_{\text {meter }}\right)$. Equation (2) in this geometry reduces to

$$
\frac{1}{r} \frac{\partial}{\partial r}(r u)+\frac{\partial w}{\partial z}=0
$$

The $r$-component of Equation (11) is

$$
\rho\left(\frac{\partial u}{\partial t}+u \frac{\partial u}{\partial r}+w \frac{\partial w}{\partial z}\right)=-\frac{\partial p}{\partial r}+\mu\left[\frac{1}{r} \frac{\partial}{\partial r}\left(r \frac{\partial u}{\partial r}\right)+\frac{\partial^{2} u}{\partial z^{2}}-\frac{u}{r^{2}}\right]
$$

while the $z$-component is

$$
\begin{aligned}
\rho\left(\frac{\partial w}{\partial t}+u \frac{\partial w}{\partial r}+w \frac{\partial w}{\partial z}\right)= & -\frac{\partial p}{\partial z}+\mu\left[\frac{1}{r} \frac{\partial}{\partial r}\left(r \frac{\partial w}{\partial r}\right)+\frac{\partial^{2} w}{\partial z^{2}}\right] \\
& -\alpha g \rho \Delta T+F_{\text {meter }}
\end{aligned}
$$

Here $\Delta T=T-T_{0}$, where $T_{0}$ is the temperature corresponding to density $\rho_{0}$. Finally, Equation (15) simplifies to

$$
\frac{\partial T}{\partial t}+u \frac{\partial T}{\partial r}+w \frac{\partial T}{\partial z}=\kappa\left[\frac{1}{r} \frac{\partial}{\partial r}\left(r \frac{\partial T}{\partial r}\right)+\frac{\partial^{2} T}{\partial z^{2}}\right],
$$

since in our case there is no internal heat source and therefore $J=0$. We therefore have four equations for four unknowns $u$, $w, T$ and $p$.

\subsection{Initial and boundary conditions}

We assume the following initial conditions

$$
\begin{aligned}
u(0, r, z) & =u_{0}(r, z), \\
w(0, r, z) & =w_{0}(r, z), \\
T(0, r, z) & =H(r, z) .
\end{aligned}
$$

We can take two different cases for the initial conditions of the velocity field; either the velocity field is zero initially and the motion is activated by the electrical pull $F_{\text {meter }}$ or by some pressure difference, or we impose non-zero initial conditions and the electrical pull further enhances the motion of the fluid.

We consider fixed temperature at the boundary,

$$
T(t, R, z)= \begin{cases}M_{1} & a \leq z \leq b \\ M_{2} & \text { otherwise }\end{cases}
$$

with radial symmetry

$$
\frac{\partial T}{\partial r}(t, 0, z)=0,
$$

where $R$ is the radius of the cylinder, and $M_{i}$ some constant temperature at the different parts of the pipe. For $M_{1}>M_{2}$ we say that the boundary condition (23) models a heater, with heating applied on $a \leq z \leq b$. One can also consider convective heat at the boundary but we do not pursue this case here.

For the velocity field, we require that no fluid passes through the wall which translates to $u=0$ and that there is no relative tangential velocity between the wall and the fluid immediately next to it, the no slip condition, which means $w=0$ at $r=R$ for all $z, t$. In the case of zero initial velocity field, we assume that there is some pressure difference $\Delta p$ between the lower and upper boundary which is driving the fluid upwards such that

$$
\begin{aligned}
& p(t, r, 0)=p_{1}+\Delta p, \\
& p(t, r, L)=p_{1} .
\end{aligned}
$$

\subsection{Non-dimensional model}

We assume the following non-dimensional quantities $t^{*}=$ $\frac{U}{R} t, r^{*}=\frac{r}{R}, z^{*}=\frac{z}{R}, u^{*}=\frac{u}{U}, w^{*}=\frac{w}{U}, p^{*}=\frac{p-p_{1}}{U^{2} \rho}, T^{*}=\frac{T-T_{0}}{\theta}$, $L^{*}=\frac{L}{R}$, where $R$ is the radius of the pipe, of characteristic travelled length $L, U$ is the characteristic speed, $\theta$ is a reference temperature difference in the flow field and $T_{0}$ is a reference temperature corresponding to density $\rho_{0}$. For simplicity we assume that in what follows $\Delta p=U^{2} \rho$. The non-dimensional numbers and parameters are $\operatorname{Re}=\frac{\rho U R}{\mu}, G r=\frac{\alpha g \theta \rho^{2} R^{3}}{\mu^{2}}, \operatorname{Pr}=\frac{\mu}{\rho \kappa}$, $\Phi=\frac{R F_{\text {meter }}}{\rho U^{2}}, M_{i}^{*}=\frac{M_{i}-T_{0}}{\theta}, a^{*}=\frac{a}{R}, b^{*}=\frac{b}{R}$.

Dropping the asterisks for convenience, the full nondimensional problem becomes

$$
\begin{gathered}
\frac{1}{r} \frac{\partial}{\partial r}(r u)+\frac{\partial w}{\partial z}=0 \\
\frac{\partial u}{\partial t}+u \frac{\partial u}{\partial r}+w \frac{\partial u}{\partial z}=-\frac{\partial p}{\partial r}+\frac{1}{\operatorname{Re}}\left[\frac{1}{r} \frac{\partial}{\partial r}\left(r \frac{\partial u}{\partial r}\right)+\frac{\partial^{2} u}{\partial z^{2}}-\frac{u}{r^{2}}\right] \\
\frac{\partial w}{\partial t}+u \frac{\partial w}{\partial r}+w \frac{\partial w}{\partial z}=-\frac{\partial p}{\partial z}+\frac{1}{\operatorname{Re}}\left[\frac{1}{r} \frac{\partial}{\partial r}\left(r \frac{\partial w}{\partial r}\right)+\frac{\partial^{2} w}{\partial z^{2}}\right]-\frac{G r}{R e^{2}} T+\Phi
\end{gathered}
$$




$$
\frac{\partial T}{\partial t}+u \frac{\partial T}{\partial r}+w \frac{\partial T}{\partial z}=\frac{1}{\operatorname{PrRe}}\left[\frac{1}{r} \frac{\partial}{\partial r}\left(r \frac{\partial T}{\partial r}\right)+\frac{\partial^{2} T}{\partial z^{2}}\right],
$$

with initial conditions

$$
u(0, r, z)=u_{0}(r, z), \quad w(0, r, z)=w_{0}(r, z), \quad T(0, r, z)=H(r, z) .
$$

For the boundary conditions for the temperature we have radial symmetry

$$
\frac{\partial T}{\partial r}(t, 0, z)=0
$$

and fixed temperature at the boundary gives

$$
T(t, 1, z)= \begin{cases}M_{1} & a \leq z \leq b \\ M_{2} & \text { otherwise }\end{cases}
$$

The velocity field boundary conditions are $u(t, 1, z)=$ $w(t, 1, z)=0$, while the pressure conditions are $p(t, r, 0)=1$ and $p(t, r, L)=0$.

\section{Numerical method}

To solve the full problem in cylindrical coordinates numerically, we will use the finite element method. To implement a finite element discretization we require the equations to be in the following form

$$
\begin{gathered}
\nabla \cdot \mathbf{u}=0 \\
\frac{\partial \mathbf{u}}{\partial t}+(\mathbf{u} \cdot \nabla) \mathbf{u}=-\nabla p+\frac{1}{\operatorname{Re}} \nabla^{2} \mathbf{u}-\frac{G r}{\operatorname{Re}^{2}} T \mathbf{e}_{2}+\mathbf{F}_{\text {meter }}, \\
\frac{\partial T}{\partial t}+(\mathbf{u} \cdot \nabla) T=\frac{1}{\operatorname{PrRe}} \nabla^{2} T,
\end{gathered}
$$

with appropriate initial and boundary conditions as described earlier. Here $\mathbf{e}_{2}$ is the unit vector in the $z$ direction.

To solve for the velocity field and pressure we will use the Chorin Projection Method [24] as follows: First, we solve the conservation of momentum equation for some intermediate velocity $\hat{\mathbf{u}}$ ignoring pressure such that

$$
\frac{\hat{\mathbf{u}}-\mathbf{u}^{n}}{\Delta t}+\left(\mathbf{u}^{n} \cdot \nabla\right) \mathbf{u}^{n}=\frac{1}{R e} \nabla^{2} \hat{\mathbf{u}}-\frac{G r}{R e^{2}} \mathbf{e}_{2} T^{n}+\mathbf{F}_{\text {meter }}\left(t_{n}\right) .
$$

Then in the projection step we want to compute $p^{n+1}$ such that

$$
\frac{\mathbf{u}^{n+1}-\hat{\mathbf{u}}}{\Delta t}=-\nabla p^{n+1} .
$$

Applying $\nabla \cdot$ to the above and using the continuity equation $\nabla$. $\mathbf{u}^{n+1}=0$ we have

$$
\frac{1}{\Delta t} \nabla \cdot \hat{\mathbf{u}}=\nabla^{2} p^{n+1}
$$

Finally, we compute the corrected velocity $\mathbf{u}^{n+1}$ such that

$$
\mathbf{u}^{n+1}=\hat{\mathbf{u}}-\Delta t \nabla p^{n+1} .
$$

Now that we have obtain the corrected velocity, we can solve for the temperature such that

$$
\frac{T^{n+1}-T^{n}}{\Delta t}+\left(\mathbf{u}^{n+1} \cdot \nabla\right) T^{n+1}=\frac{1}{\operatorname{PrRe}} \nabla^{2} T^{n+1} .
$$

We now introduce further notation in order to proceed with the finite element discretization. We denote the domain of interest by $\Omega$ with its boundary $\partial \Omega$ (the pipe in our case). Define $H^{s}(\Omega)$ as the Sobolev space with derivatives up to order $s$ in $L^{2}(\Omega)$, and let $\mathbf{L}^{2}(\Omega)=\left(L^{2}(\Omega)\right)^{3}$ and $\mathbf{H}^{s}(\Omega)=\left(H^{s}(\Omega)\right)^{3}$. Let the inner product in $L^{2}(\Omega)$ be denoted by $\langle\cdot, \cdot\rangle$. Let $\mathcal{T}_{h}$ be a shape regular triangulation of $\Omega$ of mesh size $h$. The vector and scalar finite element spaces are

$$
\begin{array}{r}
\mathbb{W}_{h}:=\left\{\mathbf{w}_{h} \in \mathbf{L}^{2}(\Omega):\left.\mathbf{v}_{h}\right|_{\tau} \in \mathcal{P}_{2}, \forall \tau \in \mathcal{T}_{h}\right\}, \\
\mathbb{V}_{h}:=\mathbb{W}_{h} \cap \mathbf{H}_{0}^{1}(\Omega), \\
\mathbb{T}_{h}:=\left\{\psi_{h} \in H_{0}^{1}(\Omega):\left.\psi_{h}\right|_{\tau} \in \mathcal{P}_{1}, \forall \tau \in \mathcal{T}_{h}\right\}, \\
\mathbb{P}_{h}:=\left\{q_{h} \in L_{0}^{2}(\Omega) \cap C^{0}(\Omega):\left.q_{h}\right|_{\tau} \in \mathcal{P}_{1}, \forall \tau \in \mathcal{T}_{h}\right\},
\end{array}
$$

where $\mathcal{P}_{1}$ and $\mathcal{P}_{2}$ are the spaces of polynomials of degree 1 and 2 respectively, on $\tau \in \mathcal{T}_{h}$.

To transform the problem into the variational form we take the inner product of Equation (37) with $\mathbf{w} \in \mathbf{L}^{2}(\Omega) \cap \mathbf{H}_{0}^{1}(\Omega)$

$$
\begin{aligned}
\frac{1}{\Delta t}\left\langle\hat{\mathbf{u}}-\mathbf{u}^{n}, \mathbf{w}\right\rangle+\left\langle\left(\mathbf{u}^{n} \cdot \nabla\right) \mathbf{u}^{n}, \mathbf{w}\right\rangle= & \frac{1}{R e}\left\langle\nabla^{2} \hat{\mathbf{u}}, \mathbf{w}\right\rangle-\frac{G r}{R e^{2}}\left\langle\mathbf{e}_{2} T^{n}, \mathbf{w}\right\rangle \\
& +\left\langle\mathbf{F}_{\text {meter }}\left(t_{n}\right), \mathbf{w}\right\rangle .
\end{aligned}
$$

By Green's First Identity

$$
\left\langle\nabla^{2} \hat{\mathbf{u}}, \mathbf{w}\right\rangle_{\Omega}=-\langle\nabla \hat{\mathbf{u}}, \nabla \mathbf{w}\rangle_{\Omega}+\langle\nabla \hat{\mathbf{u}} \cdot \mathbf{n}, \mathbf{w}\rangle_{\partial \Omega},
$$

and by imposing $\mathbf{w}=\mathbf{0}$ on the boundary $\partial \Omega$, the variational form of Equation (37) becomes

$$
\begin{aligned}
\frac{1}{\Delta t}\langle\hat{\mathbf{u}} & \left.-\mathbf{u}^{n}, \mathbf{w}\right\rangle+\left\langle\left(\mathbf{u}^{n} \cdot \nabla\right) \mathbf{u}^{n}, \mathbf{w}\right\rangle \\
& =-\frac{1}{\operatorname{Re}}\langle\nabla \hat{\mathbf{u}}, \nabla \mathbf{w}\rangle-\frac{G r}{\operatorname{Re}^{2}}\left\langle\mathbf{e}_{2} T^{n}, \mathbf{w}\right\rangle+\left\langle\mathbf{F}_{\text {meter }}\left(t_{n}\right), \mathbf{w}\right\rangle,
\end{aligned}
$$

for all $\mathbf{w} \in \mathbf{L}^{2}(\Omega) \cap \mathbf{H}_{0}^{1}(\Omega)$. Similarly taking the inner product of Equation (39) with $q \in L_{0}^{2}(\Omega) \cap C^{0}(\Omega)$

$$
\frac{1}{\Delta t}\langle\nabla \cdot \hat{\mathbf{u}}, q\rangle=\left\langle\nabla^{2} p^{n+1}, q\right\rangle
$$

which using Green's First Identity becomes

$$
\frac{1}{\Delta t}\langle\nabla \cdot \hat{\mathbf{u}}, q\rangle=-\left\langle\nabla p^{n+1}, \nabla q\right\rangle .
$$

Taking the inner product of Equation (40) with $\mathbf{w} \in \mathbf{L}^{2}(\Omega) \cap$ $\mathbf{H}_{0}^{1}(\Omega)$

$$
\left\langle\mathbf{u}^{n+1}-\hat{\mathbf{u}}, \mathbf{w}\right\rangle=-\Delta t\left\langle\nabla p^{n+1}, \mathbf{w}\right\rangle,
$$

and finally taking the inner product of Equation (41) with $\psi \in$ $H_{0}^{1}(\Omega)$

$$
\frac{1}{\Delta t}\left\langle T^{n+1}-T^{n}, \psi\right\rangle+\left\langle\left(\mathbf{u}^{n+1} \cdot \nabla\right) T^{n+1}, \psi\right\rangle=\frac{1}{\operatorname{PrRe}}\left\langle\nabla^{2} T^{n+1}, \psi\right\rangle,
$$

we obtain

$\frac{1}{\Delta t}\left\langle T^{n+1}-T^{n}, \psi\right\rangle+\left\langle\left(\mathbf{u}^{n+1} \cdot \nabla\right) T^{n+1}, \psi\right\rangle=-\frac{1}{\operatorname{Pr} R e}\left\langle\nabla T^{n+1}, \nabla \psi\right\rangle$.

Using the above, we obtain the following algorithm to recover the solution. 


\section{Algorithm}

Initializations: $\mathbf{u}_{h}^{0}, T_{h}^{0}, \Delta t$, set boundary conditions

While $t<t_{\text {final }}$

Step 1: Solve for the intermediate velocity $\hat{\mathbf{u}}_{h} \in \mathbb{V}_{h}$ for all $\mathbf{w}_{h} \in \mathbb{V}_{h}$

$$
\begin{aligned}
\frac{1}{\Delta t}\left\langle\hat{\mathbf{u}}_{h}\right. & \left.-\mathbf{u}_{h}^{n}, \mathbf{w}_{h}\right\rangle+\left\langle\left(\mathbf{u}_{h}^{n} \cdot \nabla\right) \mathbf{u}_{h}^{n}, \mathbf{w}_{h}\right\rangle \\
& =-\frac{1}{R e}\left\langle\nabla \hat{\mathbf{u}}_{h}, \nabla \mathbf{w}_{h}\right\rangle-\frac{G r}{R e^{2}}\left\langle\mathbf{e}_{2} T_{h}^{n}, \mathbf{w}_{h}\right\rangle+\left\langle\mathbf{F}_{\text {meter }}\left(t_{n}\right), \mathbf{w}_{h}\right\rangle,
\end{aligned}
$$

along with boundary conditions for the velocity $\mathbf{u}$.

Step 2: Projection Step Solve for $p_{h}^{n+1} \in \mathbb{P}_{h}$ for all $q_{h} \in \mathbb{P}_{h}$

$$
\frac{1}{\Delta t}\left\langle\nabla \cdot \hat{\mathbf{u}}_{h}, q_{h}\right\rangle=-\left\langle\nabla p_{h}^{n+1}, \nabla q_{h}\right\rangle,
$$

with boundary conditions for the pressure $p$.

Step 3: Correction Step Solve for $\mathbf{u}_{h}^{n+1} \in \mathbb{V}_{h}$ for all $\mathbf{w}_{h} \in \mathbb{V}_{h}$

$$
\left\langle\mathbf{u}_{h}^{n+1}-\hat{\mathbf{u}}_{h}, \mathbf{w}_{h}\right\rangle=-\Delta t\left\langle\nabla p_{h}^{n+1}, \mathbf{w}_{h}\right\rangle,
$$

with boundary conditions for the velocity $\mathbf{u}$.

Step 4: Solve for $T_{h}^{n+1} \in \mathbb{T}_{h}$ for all $\psi_{h} \in \mathbb{T}_{h}$

$\frac{1}{\Delta t}\left\langle T_{h}^{n+1}-T_{h}^{n}, \psi_{h}\right\rangle+\left\langle\left(\mathbf{u}_{h}^{n+1} \cdot \nabla\right) T_{h}^{n+1}, \psi_{h}\right\rangle=-\frac{1}{\operatorname{Pr} R e}\left\langle\nabla T_{h}^{n+1}, \nabla \psi_{h}\right\rangle$,

with boundary conditions for $T$.

End.

Every step in the above algorithm creates a system of linear equations $A x=b$ which is computationally costly to solve in general. For this reason we use the Krylov Solver GMRES (Generalized Minimal Residual) with preconditioning ILU (Incomplete LU), which are suitable for nonsymmetric systems, in Steps 1, 3, and 4, and the Krylov Solver CG (Conjugate Gradient) with preconditioning AGM (Algebraic Multigrid) which is suitable for symmetric systems, in Step 2 [25]. We implement the above algorithm in Fenics [26, 27] and present the results below.

\section{Results and Discussion}

We now consider numerical solutions for the problem in cylindrical geometry using the numerical method of the previous section.

We choose the nondimensional numbers to be smaller than one might take for water in order to obtain the results in suitable time. Results for a particular fluid type can be obtained using the same algorithm for the correct non-dimensional numbers using a higher number of elements and a much smaller time step in order to ensure convergence, and the results should have qualitative agreement. High performance computing and use of supercomputers might be necessary for certain parameter values that give large non-dimensional numbers. Sample results are presented in Figures 2 - 4 for the temperature and velocity magnitude. In the results presented here we take 80 points for the mesh and parameter values $\operatorname{Re}=10^{2}, \operatorname{Pr}=1$ and $G r=10^{2}$. The time step is set to $\Delta t=0.01$ and the force from
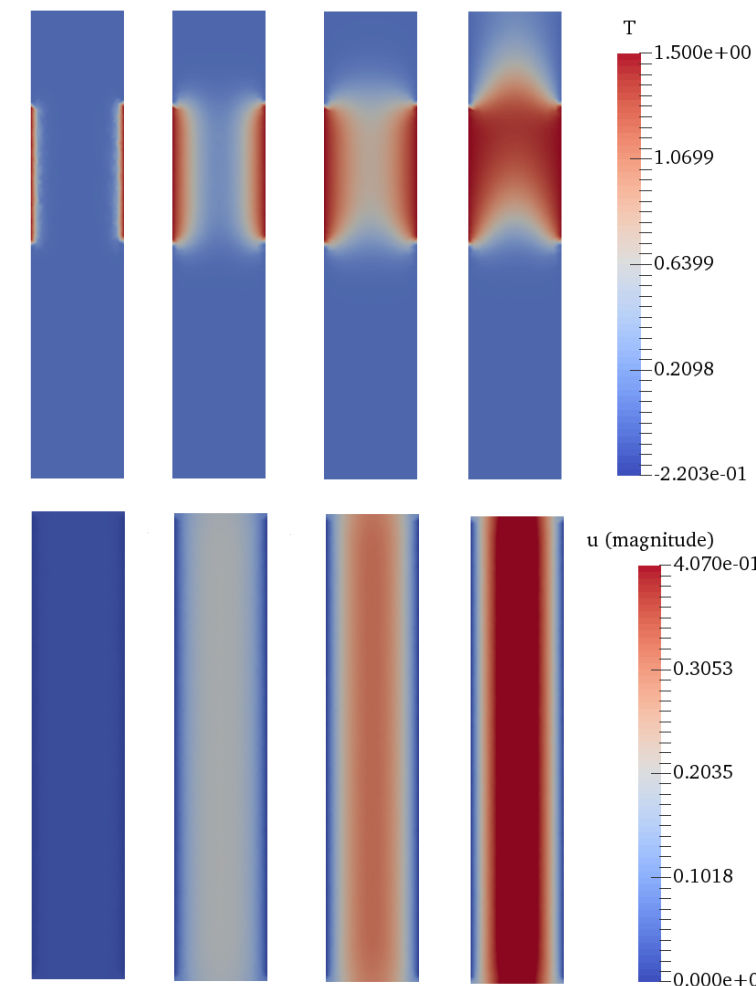

$\mathrm{t}=0$
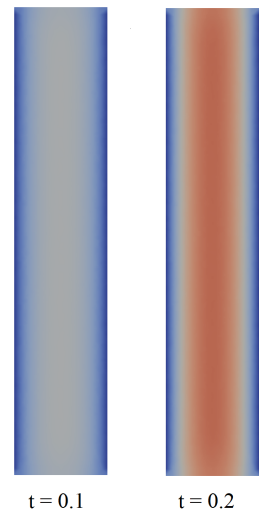

$t=0.2$

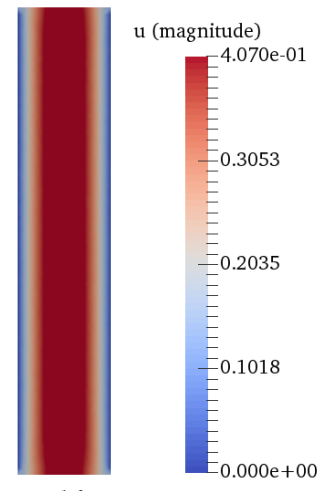

Figure 2: Numerical simulations for temperature and velocity magnitude at various non-dimensional times when the heater is applied in $0.5 \leq z \leq 0.8$.
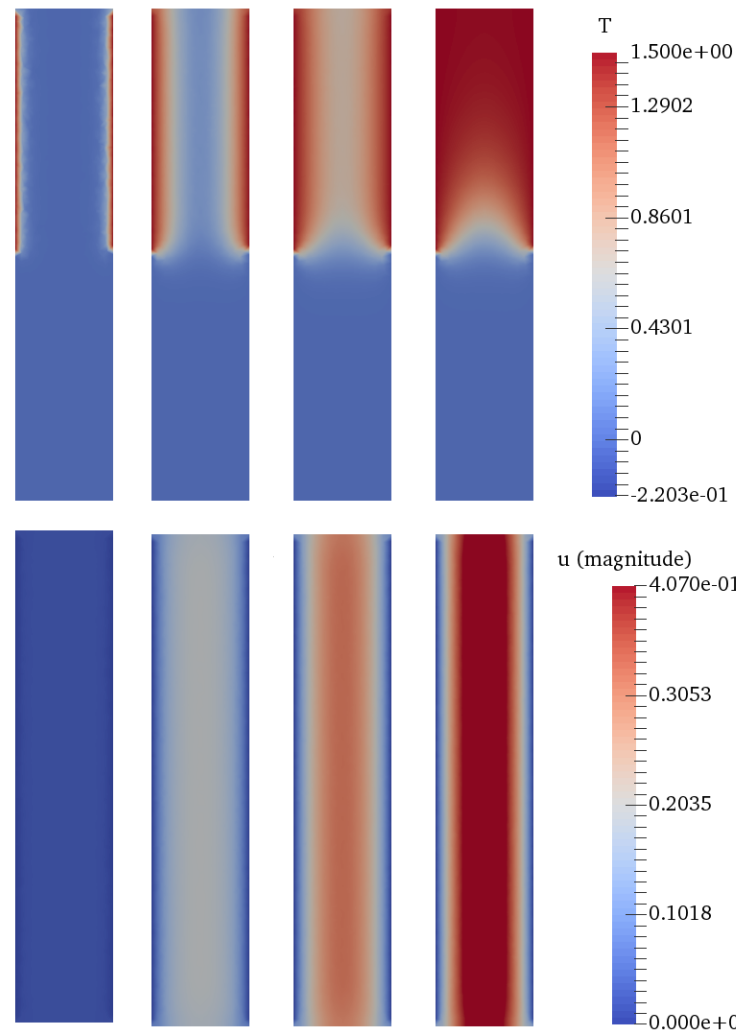

u (magnitude)

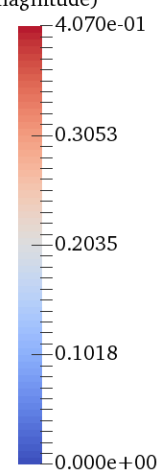

$\mathrm{t}=0$

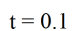

$\mathrm{t}=0.2$

$\mathrm{t}=1.0$

Figure 3: Numerical simulations for temperature and velocity magnitude at various non-dimensional times when the heater is applied on the upper half of the pipe in $0.5 \leq z \leq 1$. 

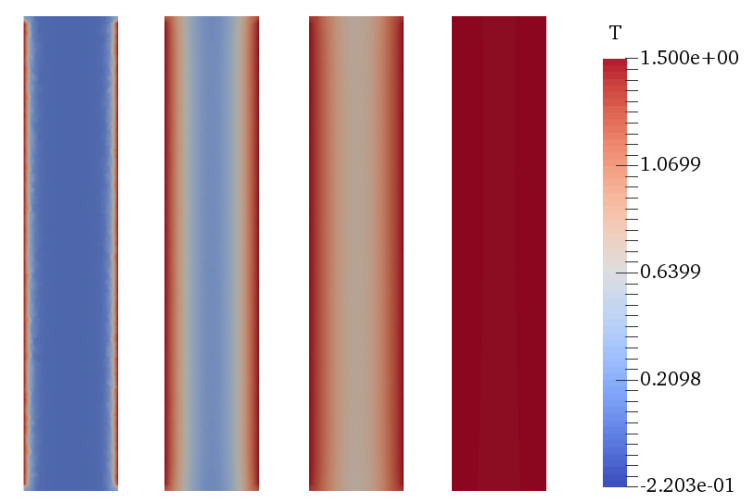

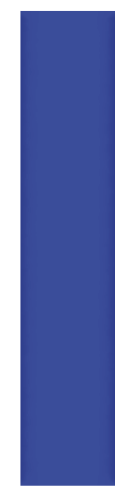

$\mathrm{t}=0$

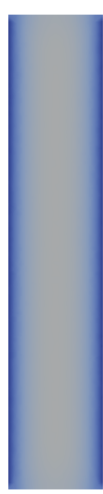

$\mathrm{t}=0.1$

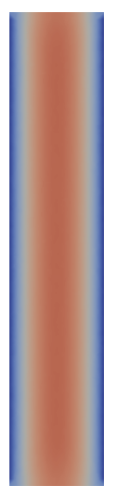

$\mathrm{t}=0.2$

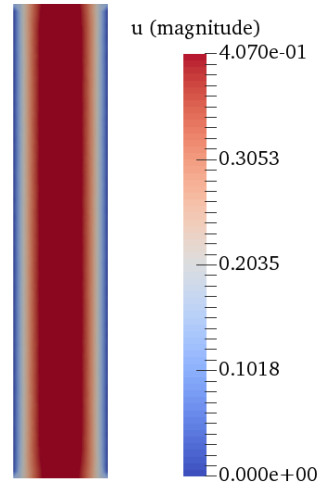

$\mathrm{t}=1.0$
Figure 4: Numerical simulations for temperature and velocity magnitude at various non-dimensional times when the heater is applied on the whole pipe.

the metre $\mathbf{F}_{\text {meter }}=(0,0,1)$ acting on the whole length of the cylinder. However, one can employ the algorithm in Section 3 to simulate the heat and mass transfer problem with parameters of relevance to ones particular application of interest.

In Figure 2 the heater is placed in the region $0.5 \leq z \leq 0.8$, in Figure 3 in the region $0.5 \leq z \leq 1$ and in Figure 4 on the full pipe. Note that we present a slice of the pipe in order to be able to observe the behaviour inside the pipe, as for the outside we prescribe constant boundary conditions. No slip boundary conditions were imposed for the velocity field with free surfaces at the top and bottom and fixed temperature at the wall, with $T=0$ representing the temperature of the pipe, and $T=1.5$ the temperature of the heater. Zero initial conditions were taken for both the velocity field and temperature. An input pressure of $p=1$ and an output pressure $p=0$ were imposed as boundary conditions. We take the length of the pipe $L=1$ and the radius $R=0.1$ and present results for time $t=0, t=0.1, t=0.2$ and $t=1$.

The results at $t=1$ appear to be steady states as any results obtained for greater times do not show any differences. The area above the heater seems to be cooling the fluid down in Figure 2 , which should be taken into account in industrial application. Some form of insulation or keeping the heater on until the end of the pipe as in Figure 3 should be considered if the required result is heated fluid at the top. Results for the pressure are not presented as they do not show any significant change with time. The velocity profile (magnitude in this case) is not affected by the temperature as we can observe similar results for all three cases.

\section{Conclusions}

We have studied the flow and temperature evolution of upstream fluid flow in a vertical pipe which is heated in some region due to an external heating element on the surface of the pipe. We have derived a heat and mass transfer model to describe the velocity, pressure, and temperature evolution in a vertical pipe. We then derive a numerical method, in particular a finite element method, which we use to provide several simulations in order to compare solutions under different heater configurations. Different outcomes were observed depending on the position and length of the heater. A partial heater that doesn't reach the top of the pipe resulted in heat loss as soon as the fluid left the heater, as one would expect. Similar results were observed in the case of the heater covering the whole length of the pipe, and a partial heater that reaches the top of the pipe, although for the latter some delay was observed until the same results were obtained. These results suggest that, so long as the desired thermal properties are attained by the time the fluid exits the top of the pipe, one may use a smaller heater covering only part of the pipe, rather than using a larger heater which covers the entire extent of the pipe. This would be much more economical, as it requires less material for the manufacture of the heater, while also requiring less energy to perform the heating. Still, one must be careful with the position of the heater as well as the size, since we found that heaters which are too small, or which are poorly placed, can result in the fluid being colder than desired at the top of the pipe.

The results we give are qualitative in nature, as we seek to demonstrate the utility of using heating elements which only cover a segment of the pipe. However, if one is interested in a particular application, then one can calibrate the model parameters accordingly and the model we provide should give reasonable results. In particular, with any set of reasonable parameter values, one could make use of the algorithm we present in Section 3 in order solve the problem with the finite element method presented. If heating is to the extent that evaporation or boiling occur (that is, the heater is replaced with a boiler), then our model would loose validity. For such flow regimes, one would need to consider a multiphase model, as we do for a boiler partially covering a pipe in [19]. In that paper we consider a onedimensional model which gives qualitatively reasonable results. It may be possible to extend such a model to a two- or -three dimensional model (as we have done in the present paper for a heater along a pipe), although we expect the combination of extra spatial dimensions with phase change boundaries would make such a model rather complicated to solve.

\section{References}

[1] Avila, K., Moxey, D., de Lozar, A., Avila, M., Barkley, D., \& Hof, B. (2011). The onset of turbulence in pipe flow. Science, 333(6039), 192196.

[2] Faghri, M., \& Sparrow, E. M. (1980). Simultaneous wall and fluid axial conduction in laminar pipe-flow heat transfer. Journal of Heat Transfer, $102(1), 58-63$. 
[3] Bilir, Ş. (1995). Laminar flow heat transfer in pipes including twodimensional wall and fluid axial conduction. International Journal of Heat and Mass Transfer, 38(9), 1619-1625.

[4] Bernier, M. A., \& Baliga, B. R. (1992). Conjugate conduction and laminar mixed convection in vertical pipes for upward flow and uniform wall heat flux. Numerical Heat Transfer, 21(3), 313-332.

[5] Zeng, H., Diao, N., \& Fang, Z. (2003). Heat transfer analysis of boreholes in vertical ground heat exchangers. International Journal of Heat and Mass Transfer, 46(23), 4467-4481.

[6] Hiroaki, T., Shigeo, M., \& Shunichi, H. (1987). Combined forced and natural convection heat transfer for upward flow in a uniformly heated, vertical pipe. International Journal of Heat and Mass Transfer, 30(1), 165174.

[7] Morton, B. R. (1960). Laminar convection in uniformly heated vertical pipes. Journal of Fluid Mechanics, 8(02), 227-240.

[8] Gupta, R. K. (1973). Unsteady laminar convection in uniformly heated vertical pipes. Journal of Fluid Mechanics, 57(01), 81-102.

[9] Barletta, A., \& Lazzari, S. (2007). Combined forced and free flow in a vertical circular duct subjected to non-axisymmetric wall heating conditions. International Journal of Heat and Mass Transfer, 50(25), 4927-4936.

[10] Smith, C. E., Faghri, M., \& Welty, J. R. (1975). On the determination of temperature distribution in laminar pipe flow with a step change in wall heat flux. Journal of Heat Transfer, 97(1), 137-139.

[11] Cai, E. Z. (2002). U.S. Patent No. 6,405,637. Washington, DC: U.S. Patent and Trademark Office.

[12] Cai, E. Z. (2007). U.S. Patent No. 7,281,467. Washington, DC: U.S. Patent and Trademark Office.

[13] Shaban, H., \& Tavoularis, S. (2014). Identification of flow regime in vertical upward air-water pipe flow using differential pressure signals and elastic maps. International Journal of Multiphase Flow, 61, 62-72.

[14] Aldridge, C. J., \& Fowler, A. C. (1992). Mathematical Modelling of Thermosyphons in Cryogenic Air Separation Plants. In Proceedings of the Sixth European Conference on Mathematics in Industry August 27-31, 1991 Limerick (pp. 75-78). Vieweg Teubner Verlag.

[15] Charoensawan, P., Khandekar, S., Groll, M., \& Terdtoon, P. (2003). Closed loop pulsating heat pipes: Part A: parametric experimental investigations. Applied Thermal Engineering, 23(16), 2009-2020.

[16] Xuan, Y., \& Li, Q. (2003). Investigation on convective heat transfer and flow features of nanofluids. Journal of Heat transfer, 125(1), 151-155.

[17] Fotukian, S. M., \& Esfahany, M. N. (2010). Experimental investigation of turbulent convective heat transfer of dilute $\gamma$-Al $2 \mathrm{O}$ 3/water nanofluid inside a circular tube. International Journal of Heat and Fluid Flow, 31(4), 606-612.

[18] Fotukian, S. M., \& Esfahany, M. N. (2010). Experimental study of turbulent convective heat transfer and pressure drop of dilute $\mathrm{CuO} /$ water nanofluid inside a circular tube. International Communications in Heat and Mass Transfer, 37(2), 214-219.

[19] Kleanthous, A., \& Van Gorder, R. A. (2016). Influence of boiler size and location on two-phase vertical pipe flows, preprint.

[20] Boussinesq, M. J. (1897). Thèorie de l'ècoulement tourbillonnant et tumultueux des liquides. Gauthier-Villars.

[21] Tritton, D. J. (2012). Physical fluid dynamics. Springer Science \& Business Media.

[22] Quarteroni, A., Sacco, R., \& Saleri, F. (2010). Numerical mathematics (Vol. 37). Springer Science \& Business Media.

[23] Fornberg, B. (1988). Generation of finite difference formulas on arbitrarily spaced grids. Mathematics of Computation, 51(184), 699-706.

[24] Chorin, A. J. (1967). The numerical solution of the Navier-Stokes equations for an incompressible fluid. Bulletin of the American Mathematical Society, 73(6), 928-931.

[25] Benzi, M. (2002). Preconditioning techniques for large linear systems: a survey. Journal of Computational Physics, 182(2), 418-477.

[26] Alnaes M. S., Blechta J., Hake J., Johansson A., Kehlet B., Logg A., Richardson C., Ring J., Rognes M. E. \& Wells G. N. (2015). The FEniCS Project Version 1.5. Archive of Numerical Software, 3(100), [doi:10.11588/ans.2015.100.20553].

[27] Logg A., Mardal K.-A., Wells G. N. et al. (2012). Automated Solution of Differential Equations by the Finite Element Method, Springer. [doi:10.1007/978-3-642-23099-8] 